\title{
Droits de l'homme et insecurité pendant le processus de démocratisation en République Démocratique du Congo: Cas de la ville de Lubumbashi
}

\author{
Martial MUMBA KAKUDJI*
}

Resumé: La République Démocratique du Congo, aux termes de la Constitution du 18 fevrier 2006, est un Etat de droit et démocratique. L'Etat de droit présuppose l'application de lois, le respect des droits de l'homme et de la démocratie. Mais, cette conception souffre de sa mise en pratique. Ceci s'explique notamment, par le fait que le processus électoral de 2018 en République Démocratique du Congo a été emaillé de certains cas graves de violations des droits de l'homme voilées par l'insécurité dans la ville de Lubumbashi. Pourtant, ces libertés et droits fondamentaux ont un caractère juridique obligatoire depuis la Déclaration universelle des droits de l'homme de 1948 et bien d'autres instruments juridiques internationaux relatifs aux droits de l'homme déjà ratifiés par la République Démocratique du Congo. Ces droits et libertés sont censés être appliqués effectivement dans tout Etat qui se veut Etat de droit et engagé dans le chemin de la démocratie. Et donc, le processus de démocratisation ne peut constituer en rien un obstacle pour leur application. C'est plutôt un moment propice de faire valoir la capacité de les appliquer. Notons quand meêm que ces violations sont à mettre à l'actif de la majorité comme de l'opposition.

\section{INTRODUCTION}

Il est connu de tous que la République Démocratique du Congo se veut un Etat de droit, c'est-à-dire un Etat dans lequel il y a prééminence du droit sur le pouvoir politique et que tous, gouvernants et gouvernés doivent obéir à la loi.

En effet, l'Etat de droit peut se définir comme un système institutionnel dans lequel la puissance publique est soumise au droit. Cette notion, d'origine allemande (Rechtsstaat), a été redéfinie au début du vingtième siècle par le juriste autrichien Hans Kelsen, comme un Etat dans lequel les normes juridiques sont hiérarchisées de telle sorte que sa puissance s'en trouve limitée. Dans ce modèle, chaque règle tire sa validité de sa conformité aux règles supérieures. Un tel système suppose, par ailleurs, l'égalité des sujets de droit devant les normes juridiques et l'existence de juridictions indépendantes.

Il convient d'indiquer que l'existence d'une hiérarchie des normes constitue l'une des plus importantes garanties de l'Etat de droit. Dans ce cadre, les compétences des différents organes de l'Etat sont précisément définies et les normes qu'ils édictent ne sont valables qu'à condition de respecter l'ensemble des normes de droit supérieures. Au sommet de cet

* Chef de Travaux à la Faculté de Droit de l'Université de LUBUMBASHI et Diplômé d'Etudes Approfondies en Droit public (Master). Il est Doctorant et aussi Avocat depuis 2010 au Barreau près la Cour d'Appel de Lubumbashi (martialkakudji@yahoo.fr). 
ensemble pyramidal figure la Constitution, suivie des engagements internationaux, de la loi, puis des règlements. A la base de la pyramide figurent les décisions administratives ou les conventions entre personnes de droit privé. Cet ordonnancement juridique s'impose à l'ensemble des personnes juridiques. L'Etat, pas plus qu'un particulier, ne peut ainsi méconnaître le principe de légalité : toute norme, toute décision qui ne respecteraient pas un principe supérieur seraient susceptibles d'encourir une sanction juridique.

L'Etat, qui a compétence pour édicter le droit, se trouve ainsi lui-même soumis aux règles juridiques, dont la fonction de régulation est ainsi affirmée et légitimée. Un tel modèle suppose donc la reconnaissance d'une égalité des différents sujets de droit soumis aux normes en vigueur ${ }^{1}$.

L'égalité des sujets de droit constitue sans doute la deuxième condition de l'existence d'un Etat de droit. Celui-ci implique que tout individu, toute organisation, puissent contester l'application d'une norme juridique, dès lors que cette dernière n'est pas conforme à une norme supérieure. Les individus et les organisations reçoivent en conséquence la qualité de personne juridique : on parle de personne physique dans le premier cas, de personne morale, dans le second.

Cette conception est d'une manière ou d'une autre reprise dans la Constitution du 18 février 2006 telle que modifiée et complétée à ce jour par la loi $\mathrm{N}^{\circ} 11 / 002$ du 20 janvier 2011 portant révision de certains articles de la Constitution lorsqu'elle celle-ci stipule en son article premier, alinéa 1 ce qui suit : «La République Démocratique du Congo est, dans ses frontières du 30 juin 1960, un Etat de droit, indépendant, souverain, uni et indivisible, social, démocratique et laïc $»$.

Cette Constitution consacre en même temps tout un titre aux droits humains, aux libertés fondamentales et aux droits du citoyen et de l'Etat. Ceci signifie autrement la reconnaissance des droits de l'homme dans un Etat de droit qui implique aussi le respect et l'application de ces droits fondamentaux.

Dans un Etat de droit, le respect des droits de l'homme et de la démocratie constitue le soubassement de cet Etat. A propos, Norberto Bobbio offre une précieuse clé d'entrée pour penser les rapports entre la démocratie, la multiplication des droits et l'affirmation de contre-pouvoirs. Bien que ce dernier affirme soutenir, dans la continuité de Hans Kelsen, une définition formelle ou procédurale de la démocratie en la réduisant à un ensemble de règles du jeu qui permettent d'adopter, avec le plus large consentement de leurs destinataires, des décisions collectives sans déterminer leur contenu politique, économique ou social, il lie aussi consubstantiellement la démocratie à un ensemble de valeurs.

Figurent au premier rang de celles-ci, la paix et les droits de l'homme : « Droits de l'homme, démocratie, et paix sont les trois moments nécessaires d'un même mouvement historique : sans droits de l'homme reconnus et effectivement protégés, il n'y a pas de démocratie; sans démocratie, il n'y a pas les conditions minimales pour la résolution pacifique

1 https://www.vie-publique.fr/decouverte-institutions/institutions/approfondissements/qu-est-ce-que-e tat-droit.html, page consultée le 06 juin 2019. 
des conflits ${ }^{2}$. Il s'agit en fait de l'interdépendance des droits de l'homme et de la démocratie.

S'agissant des droits de l'homme, il y a lieu de noter que les droits de l'homme ne sont pas une catégorie intemporelle. Ils ont émergé, à l'issue d'une lente maturation de la pensée politique et philosophique, à une époque et en un lieu donnés : l'Europe du XVIIème et du XVIIIème siècle. Proclamés solennellement en 1789 par la Déclaration des droits de l'homme et du citoyen, inscrits d'abord dans les textes, ils se sont peu à peu ancrés dans les mentalités, dans les pratiques sociales. Cette extension progressive a été concomitante de l'installation et de l'affermissement des régimes démocratiques. Extension incomplète, bien sûr, et fragile de surcroît, comme l'ont montré, dans la première moitié du XXème siècle, le retour des dictatures, l'expérience des régimes totalitaires, les atrocités de la Seconde Guerre mondiale.

La Déclaration universelle de 1948 a marqué une nouvelle étape dans l'histoire des droits de l'homme, désormais placés sous la protection de la communauté internationale.

Si cette prise en charge institutionnelle n'a pas suffi à instaurer le règne sans partage des droits de l'homme sur la planète, elle a pu contribuer à en donner une vision pacifiée et consensuelle. Il a fallu attendre les années 1970 pour qu'ils retrouvent la dimension contestataire et subversive qui était la leur en 1789 et qui s'était progressivement estompée, soit parce qu'ils paraissaient chose acquise, soit parce que la marche vers un avenir radieux semblait devoir emprunter d'autres voies ${ }^{3}$.

En tout état de cause, la Déclaration Universelle des Droits de l'homme, bien qu'elle ne soit pas contraignante, est une référence. Elle a fait l'objet de plusieurs traductions. Tous les ans, le 10 décembre, le monde célèbre son adoption; c'est la journée des droits de l'homme. La plupart des accords conclus dans ce sillon, notamment des Pactes sur les droits civils et politiques, sur les droits économiques, sociaux et culturels ou de la Convention relative aux droits de l'enfant, créent des comités chargés de suivre leur application, dont les membres sont élus par les Etats parties à ces accords ${ }^{4}$.

Les droits de l'homme constituent l'ensemble des droits qui conditionnent à la fois la liberté de l'Homme, sa dignité et l'épanouissement de sa personnalité» ${ }^{5}$. C'est l'ensemble des droits qui permettent de préserver la dignité de l'Homme et lui permettent de se réaliser et de vivre sa personne, de s'accomplir. Leur objet est par conséquent directement et intimement lié à la liberté des personnes et au respect de leur dignité humaine sans nulle autre raison ou fondement.

2 VERONIQUE CHAMPEIL-DESPLATS, « Démocratie, droits de l'homme et contrôle de constitutionnalité chez Norberto Bobbio ", in La démocratie, entre multiplication des droits et contre-pouvoirs sociaux, éditions Kimé, collection : Nomos et Normes, 2012, pp.25 - 40.

3 DANIEL LOCHAK, Les droits de l'homme, éditions La Découverte, Collection : Repères, 2018, pp.3 -7.

4 JEAN-MARC DE LA SABLIERE, Indispensable ONU, éditions PILON, Paris, 2017, p.77.

5 ROCHE (Jean) et POUILLE (André), Libertés publiques et droits de l'Homme, 13èmeédition, 1999, p.6. 
Trois remarques doivent être formulées à ce niveau concernant cette définition. Premièrement, et malgré la clarté de cette définition, le contenu des droits de l'Homme ne fait pas l'unanimité car la notion de dignité humaine varie suivant les époques, les cultures et les conceptions. Les droits de l'Homme seront envisagés dans ce cours d'un point de vue déterminé, celui de la conception onusienne. Deuxièmement, et du point de vue de leur nature, les droits de l'Homme se subdivisent en deux grandes catégories: des « droits à » et des « droits de ». Les " droits de » sont les droits de faire quelque chose, des droits actifs en quelque sorte (droit de grève, droit de circuler, droit de s'exprimer, droit de s'associer, droit de manifester...). Alors que les «droits à » sont des droits à l'obtention de quelque chose, des droits passifs d'un certain point de vue (droit à la santé, droit à l'éducation, droit à l'intégrité physique, droit à la sûreté...).Troisièmement, une distinction importante doit être faite entre droits de l'Homme et libertés publiques.

Les libertés publiques peuvent, en effet, être définies comme des pouvoirs d'autodétermination, reconnus et organisés par l'Etat, par lesquels l'Homme choisit lui-même son comportement $^{6}$ (leur caractère public faisant référence à leur inscription et à leur garantie par le Droit positif et non pas à leur utilisation par plusieurs personnes; ces libertés peuvent en effet être individuelles c'est-à-dire exercées par chaque personne individuellement, ou collectives). Tel que définis précédemment, les droits de l'Homme ne se ramènent pas seulement à la revendication ou à l'exercice d'une liberté; d'autres droits dont l'être humain jouit lui permettent d'exiger de la société la satisfaction de ses besoins vitaux tels que le droit au travail, à la sécurité sociale, à la santé, à la culture ou à l'instruction...etc. Dans ces derniers cas par exemple, il s'agit bien de droits faisant partie des droits de l'Homme sans qu'il ne s'agisse de libertés; " leur reconnaissance par le droit positif donne à l'Homme un pouvoir d'exiger une créance, mais ne fonde pas une liberté publique» ${ }^{7}$. La notion de droits de l'Homme englobe ainsi celle des libertés publiques qui n'en sont qu'un aspect parmi d'autres. D'autres droits tels que le droit à la paix, au développement durable, à l'égalité, à la non-discrimination, à un environnement sain et équilibré, font partie intégrante des droits de l'Homme mais ne sont pas des libertés.

En effet, dans le cadre de cette réflexion, il sied de préciser que cette analyse va se focaliser sur la première et la deuxième catégorie des droits de l'homme pour ne pas parler de la première et deuxième génération des droits de l'homme, à savoir les droits civils et politiques et les droits sociaux. Les droits de l'homme, malgré cette différenciation en termes de génération, restent interdépendants les uns des autres.

Par ailleurs, depuis un certain temps, République Démocratique du Congo et d'une manière particulière la ville de Lubumbashi se caractère par une forme d'insécurité qui ne dit pas son nom alors qu'il s'agit d'un Etat qui se veut Etat de droit et engagé dans le processus de démocratisation qui présuppose le respect et l'application des droits de l'homme.

6 RIVERO JEAN, Libertés publiques, Manuel, éditions PUF, 1973, pp.16 - 17.

7 Idem, p.17. 
L'insécurité est à la base de beaucoup de cas des violations des droits de l'homme au point que nous la considérons comme étant une autre face des violations des droits de l'homme. Et pourtant, ces droits ne doivent pas souffrir d'application quelle que soit la période que traverse l'Etat congolais.

En principe, c'est pendant cette période de processus de démocratisation que la République Démocratique du Congo devrait se montrer déterminée, pour ne pas dire un bon élève en appliquant les droits de l'homme si elle se veut vraiment un Etat de droit et démocratique.

De par ce constat déplorable, il y a lieu de se poser une série de questions à savoir si le processus de démocratisation constitue-t-il un obstacle à l'application effective des droits de l'homme en République Démocratique du Congo; comment expliquer cette recrudescence de l'insécurité et quels sont les solutions à proposer pour que l'insécurité cède la place à la sécurité sécurisante.

Ces préoccupions permettent de circonscrire cette analyse et poussent à chercher et à donner des pistes de solutions en rapport avec la réalité sur terrain. Pour mener à bon port cette étude, le recours à la méthode systémique est d'un grand apport. Elle a le mérite de viser à découvrir les lois d'arrangement de la réalité sociale considérée comme un ensemble organisé et ordonné ${ }^{8}$. Cette méthode est globale et globalisante en ce qu'elle permet de bien cerner l'objet à partir de quelques principes aux lois ci-après :

- Le principe de globalité; les phénomènes sociaux, totaux et globaux considérés dans leur totalité pour éviter le décalage, le respect des règles et des événements constituant un système global, l'absence de l'un pouvait constituer un déséquilibre dans le système tout entier;

- Le principe d'interdépendance des éléments du système : les éléments du système sont examinés en fonction d'autres éléments qui composent celui-ci;

- Le principe d'autorégulation, d'organisation du changement au sein du système.

Cette méthode permet de comprendre les déséquilibres dans l'application de la législation congolaise en ce qui concerne l'application et le respect des droits de l'homme. et donc, cette méthode part de l'hypothèse que le droit est ordonné et interdépendant et que les règles de droit peuvent être interprétées en fonction du contexte dans lequel elles se situent ${ }^{9}$.

En plus de cette méthode, il appert important de faire aussi recours à une approche sociojuridique qui a le mérite d'interpréter les dispositions constitutionnelles et d'autres lois relatives aux droits de l'homme qui doivent s'appliquer dans la société congolaise.

8 ANDRIEN MULUMBATI NGASHA, Manuel de sociologie générale, Lubumbashi, éditions Africa, 2010, pp.24-25.

9 PIERRE FELIX KANDOLO ON'UFUKU WA KANDOLO, Guide Kandolo : Méthodes et règles de rédaction d'un travail de recherche en droit, éditions Universitaires Européennes, Mauritius, 2018, p.314. 
Outre les méthodes utilisées, il est fait usage de la technique documentaire et d'observation directe ainsi qu'aux réseaux sociaux pour la concrétisation de méthodes employées.

L'objet de cette étude est de décrier d'une part l'insécurité dont la population congolaise en général et lushoise en particulier est victime alors qu'elle est censée être sécurisée par l'Etat à qui incombe la responsabilité de protéger et de sécuriser les personnes et leurs biens L'insécurité est à la base de violations des droits de l'homme pendant le processus de démocratisation présupposant en principe le respect de ces droits se pratique par l'utilisation des méthodes. Mais d'autre part, de dénoncer les méthodes utilisées pour violés les droits de l'homme suite à l'insécurité.

\section{A. CONSECARTION CONSTITUTIONNELLE DES DROITS DE L'HOMME EN REPUBLIQUE DEMOCRATIQUE DU CONGO}

\section{Les droits de l'homme dans le contexte congolais}

La conception des droits de l'homme a évolué au point qu'ils ont été qualifiés des droits universels, repris comme tels dans plusieurs instruments juridiques internationaux et intégrés dans les constitutions des Etats. Toujours préoccupés par la protection de la dignité de la vie de l'homme et de ses droits, la Communauté internationale ${ }^{10}$ n'a cessé d'interpeler les Etats à respecter ces droits. L'expression des droits de l'homme semble être toute récente alors qu'elle était déjà d'actualité dans l'Antiquité ${ }^{11}$. Les droits de l'homme sont devenus une préoccupation universelle. Il n'y a plus d'organisation politique dans le monde qui ne prétende donner à son action les droits de l'homme.

L'Organisation des Nations Unies, dans le cadre des droits de l'homme, a toujours déployé des activités pour la promotion et le respect de ces droits à tel enseigne qu'elle a permis aux Etats d'être attentifs à la question des droits de l'homme.

La République Démocratique du Congo, comme d'autres Etats, n'est pas restée à l'écart, elle s'est aussi efforcée de s'adapter aux impératifs de l'Organisation des Nations

10 RENE-JEAN DUPUY, à l'évidence, affirme à propos de ce concept qu'il y a nombre d'années, l'expression "Communauté internationale » a une force politique et un contenu de solidarité et de quête de justice au plan international, qui fait défaut à l'expression « Société internationale ». De là vient la préférence de plus en plus marquée pour l'utilisation de la première. Cette expression est employée de manière réitérée par la Cour internationale de justice. Son utilisation suppose une communauté globale qui ne se réduit pas à une société d'Etats considérée à un moment historique donné. Il s'agit d'une communauté complexe et multiple, constituée par des entités étatiques et non étatiques, par des personnes juridiques internationales. En outre, par des individus de l'espèce humaine, c'est-à-dire par ces personnes qui constituent ce nouveau sujet du Droit international contemporain. Cf. Federico Mayor, René-Jean Dupuy une œuvre au service de l'humanité, UNESCO, Paris, 1999, p.27.

11 CESAR NKUKU KHONDE (dir.), «Droits de l'homme au Congo colonial : exposé et analyse de quelques faits et témoignages des abus ", La République Démocratique du Congo : Les droits humains, les conflits et la construction/destruction de l'Etat, édition Fondacio Solidaritat UB et Inrevés, Barcelone, 2009, p.12. 
Unies et des Organisations régionales sur les droits de l'homme. C'est ainsi qu'elle a adhéré à plusieurs Conventions y relatives en les ratifiant et en les intégrant dans son arsenal juridique.

La Constitution de la République Démocratique du Congo telle modifiée et complétée à ce jour par la loi $\mathrm{n}^{\circ} 11 / 002$ du 20 janvier 2011 portant révision de certains articles de la Constitution de la République Démocratique du Congo du 18 février 2006 consacre les droits humains, les libertés fondamentales et les devoirs du citoyen et de l'Etat.

La première catégorie de ces droits consacre l'égalité sous toutes ses formes y compris, naturellement, entre l'homme et la femme; les garanties de la personne, à savoir le droit à la vie, le droit à l'intégrité physique et au développement de la personnalité, l'interdiction de l'esclavage, des traitements cruels, inhumains ou dégradants et du travail forcé; les garanties d'un procès équitable et de la procédure judiciaire; la liberté de pensée, de conscience et de religion; la liberté d'expression; le droit à l'information qui implique, expressément, la liberté de la presse; la liberté de réunion, de manifestation, de pétition; la liberté de circulation et d'établissement sur le territoire national; le droit au respect de la vie privée; le droit d'asile etc.... ${ }^{12}$

La deuxième catégorie quant à elle reconnait la propriété privée, le droit à l'initiative privée; le droit au travail et à la protection sociale; la liberté d'association, la liberté syndicale, le droit de grève; le droit à l'éducation scolaire et notamment à un enseignement primaire obligatoire et gratuit dans les établissements publics; le droit à la culture, le droit à un environnement sain et le droit pour tous les congolais de pouvoir jouir des richesses nationales ${ }^{13}$.

De tout ce qui précède, il y a lieu de comprendre que l'un des objectifs du constituant congolais, de par le préambule et le titre deux de la Constitution, a été de mettre en place un Etat démocratique et, plus généralement, de garantir les droits des citoyens.

Il y a lieu de reconnaitre que le constituant congolais accorde une place importante à la garantie des droits et libertés. Le titre deuxième de la Constitution, composé de 57 articles contient, en effet, une Déclaration des droits « complète, moderne et adaptée aux préoccupations actuelles de la société congolaise ». Il ne se contente néanmoins pas de proclamer ces droits, il souhaite les garantir. De ce fait, c'est au pouvoir judiciaire que revient cette mission conformément à l'article 150, alinéa 1 de la Constitution qui dispose que : «Le pouvoir judiciaire est le garant des libertés individuelles et des droits fondamentaux des citoyens ».

Ce pouvoir comprend notamment, la Cour constitutionnelle qui doit jouer un rôle déterminant en tant que gardienne de la Constitution et de l'équilibre des pouvoirs. En fait, au-

12 Articles 11 à 33 de la Constitution du de la République Démocratique du Congo telle que modifiée et complétée par la Loi $\mathrm{n}^{\circ} 11 / 002$ du 20 janvier 2011 portant révision de certains articles de la Constitution de la République Démocratique du Congo.

13 Articles 34 à 57 de la Constitution du de la République Démocratique du Congo telle que modifiée et complétée par la Loi $\mathrm{n}^{\circ} 11 / 002$ du 20 janvier 2011 portant révision de certains articles de la Constitution de la République Démocratique du Congo. 
delà des droits et libertés, c'est en réalité l'Etat de droit que doit garantir la Cour constitutionnelle, Etat de droit dont la préservation reste l'objectif primordial du constituant congolais ${ }^{14}$. L'aménagement juridique des droits de l'homme en Droit positif congolais fait apparaître la dialectique entre l'intérêt de la sauvegarde des droits de l'individu et les nécessités liées à la préservation de l'intérêt général. Dans tous les cas, malgré les volontés politiques contraires, les restrictions et les dérogations voulues aux droits de l'homme aboutissant, au final, à la réaffirmation de leur principe, à savoir qu'il n'est pas permis de violer impunément les droits et prérogatives reconnues à la personne humaine ${ }^{15}$.

\section{Les mécanismes de protection et de mise en œuvre}

Le respect de la dignité et de la valeur humaine constitue la substance des droits de l'homme. Les mécanismes de protection et de mise en œuvre sont prévus tant au niveau international, régional que national. Mais ce qui est étonnant, c'est le fait que les droits de l'homme continuent toujours d'être violés.

Il y a lieu de noter qu'il existe des mécanismes conventionnels et non conventionnels. Dans le système des Nations Unies, les mécanismes sont de pourvus de caractère juridictionnel contraignant en dépit de leur portée politique et morale indéniable ${ }^{16}$.

En effet, leur acceptabilité et leur application directe sont tributaires de la souveraineté des Etats et de leur acceptation expresse des obligations qui en découlent.

Comme presque tous les problèmes humains ont un aspect qui intéresse les droits de l'homme, tous les principaux organes des Nations Unies à savoir l'Assemblée générale, le Conseil de sécurité, le Conseil de tutelle, la Cour internationale de justice et le Conseil économique et social; abordent d'une façon ou d'une autre la question des droits de l'homme A ces organes, il faut y ajouter certains organismes subsidiaires comme le Haut-Commissariat des Nations Unies aux Droits de l'homme et les structures opérationnelles comme le Bureau Conjoint des Nations Unies aux droits de l'homme.

Toutefois, l'Assemblée générale est le principal organe responsable des questions relatives aux droits de l'homme. Elle est investie, par la Charte des Nations Unies, en son article 13, du pouvoir de provoquer des études et de faire des recommandations en vue de " développer la coopération internationale dans les domaines économique, social et culturel, et dans celui de l'éducation et de la santé publique, afin de faciliter pour tous, sans distinction de race, de sexe, de langue ou de religion, la jouissance des droits de l'homme et des libertés fondamentales ».

14 DELPHINE POLLET-PANOUSSIS, « La Constitution congolaise de 2006 : petite sœur africaine de la Constitution française », in Revue française de droit constitutionnel, Presses Universitaires de France, $2008 / 3$ n $^{\circ} 75$, p.488. Disponible en ligne à l'adresse : https://www.cairn.info/revue-francais e-de-droit-constitutionnnel-2008-3-page-451.htm.

15 NGONDANKOY NKOY-EA-LOONGYA, Droit congolais des droits, éditions Academia-Bruylant, Paris, 2004, p.489.

16 Idem, p.335. 
Sur cette base, l'Assemblée générale dispose de pouvoirs décisionnels et remplit un rôle de coordination. C'est ainsi qu'elle est amenée à adopter les textes normatifs et à les ouvrir à la signature des Etats $^{17}$.

La majeure partie des travaux de l'Assemblée générale s'effectue en commission et c'est à la Troisième commission, chargée des questions sociales, humanitaires et culturelles, qu'incombe la responsabilité de traiter des questions relatives aux droits de l'homme. Mais étant donné l'étendue des activités de l'Assemblée générale, l'article 62 de la Charte a naturellement délégué au Conseil économique et social le pouvoir de faire des recommandations afin d'assurer le respect des droits de l'homme et des libertés fondamentales pour tous. Il peut également préparer des projets de Convention pour les soumettre à l'Assemblée générale. Enfin, en vertu de l'article 62 de la Charte, il dispose de la possibilité d'instituer des commissions dans ce domaine. Il a ainsi créé la Commission des droits de l'homme actuellement le conseil des droits de l'homme. Il est important de noter que le conseil des droits de l'homme est un organe politique composé de représentants des Etats qui agissent sur leurs instructions. Il est constitué des Etats membres choisis pour trois ans en fonction de subtils équilibres régionaux pour donner une image fidèle de la communauté internationale ${ }^{18}$.

En pratique, ses activités ont rapidement débordé le cadre strict des études, pour s'étendre à la protection des droits de l'homme sous tous ses aspects.

Ce travail n'a pas la prétention d'entrer en détails en ce qui concerne les autres mécanismes de protection prévus par d'autres systèmes, il se veut plus développer les mécanismes prévus en République Démocratique du Congo pour la mise en œuvre des instruments juridiques relatifs aux droits de l'homme.

Certes, la République Démocratique du Congo dispose de ses propres mécanismes qui coexistent avec les mécanismes régionaux et internationaux en vigueur, mais elle patauge à ce jour autour de l'organisation ou de la possibilité d'organisation des structures et des mécanismes aussi divers que variés, dont l'objectif final demeure l'atteinte d'un meilleur niveau de protection des droits de l'homme ${ }^{19}$.

La Constitution de la République Démocratique du Congo prévoit les mécanismes notamment juridictionnels de la mise en œuvre des instruments juridiques relatifs aux droits de l'homme. L'article 150 de la Constitution de la République Démocratique du Congo telle

17 FABRICE PARFAIT OUMBA, Les mécanismes de contrôle et de garantie des droits de l'homme, p.3. Disponible sur https://hal.archives-ouvertes.fr/cel-01319645, Submitted on 21 may 2016, page consultée le 05/06/2019.

18 RUSEN ERGEC, Protection européenne et internationale des droits de l'homme, Bruxelles, Bruylant, 2004, p.59.

19 PIERRE-FELIX KANDOLO ON'UFUKU WA KANDOLO, De l'exercice des droits et libertés individuels et collectifs comme garantie d'une bonne gouvernance en Afrique noire : Cas de la République Démocratique du Congo, Université de Nantes/Université de Lubumbashi, Campus numériques, $3^{{ }^{\mathrm{m} e}}$ cycle des Droits fondamentaux. Disponible sur https://www.mémoireonliine.com, page consultée le 02 juin 2019. 
que modifiée et complétée à ce jour par la loi n¹1/002 du 20 janvier 2011 portant révision de certains articles de la Constitution du 18 février 2006 dispose que : « Le pouvoir judiciaire est le garant des libertés individuelles et des droits de fondamentaux des citoyens $»^{20}$.

Il ressort clairement de cette disposition que non seulement le constituant congolais affirme que c'est au pouvoir judiciaire que revient cette mission de garantir les droits et libertés mais aussi il accorde en même temps une place importante à ces droits. C'est au sein du pouvoir judiciaire, la Cour constitutionnelle en tant que gardienne de la Constitution et de l'équilibre des pouvoirs joue un rôle déterminant. En fait, au-delà des droits et libertés, c'est en réalité l'Etat de droit que doit garantir la Cour constitutionnelle, Etat de droit dont la préservation reste l'objectif primordial du constituant congolais.

Les mécanismes de protection des droits de l'homme prévus par la République Démocratique du Congo sont entre autres les cours et tribunaux dont la Cour constitutionnelle est garante des droits fondamentaux, le Ministère des droits humains, la Commission Nationale des droits de l'homme ${ }^{21}$, la Société civile. A ces mécanismes, il convient d'ajouter les institutions d'appui ${ }^{22}$ à la démocratie dont notamment, la Commission électorale nationale indépendante et le Conseil supérieur de l'audiovisuel et de la communication qui jouent un rôle non négligeable de protection et de dénonciation de violations des droits de l'homme.

La réalisation des droits de l'homme fait référence à leur mise en œuvre. A noter que les termes peuvent fluctuer : pour parler de leur réalisation, il est parfois fait mention de concrétisation, de mise en œuvre ou de spécification des droits de l'homme.

La simple existence d'un droit de l'homme ne garantit pas qu'il soit réalisé, cependant. Au contraire, l'existence d'un droit de l'homme génère un droit à ce qu'il le soit et une obligation positive morale et légale correspondante de le mettre en œuvre. Ces obligations de réalisation appellent des mesures individuelles mais aussi structurelles et institutionnelles dans certains cas. A propos, les acteurs des droits de l'homme parlent d'ailleurs souvent d'institutionnalisation des droits de l'homme pour signifier leur réalisation. ${ }^{23}$ Les obligations de réalisation doivent être spécifiées et déterminées dans chaque cas en fonction des circonstances concrètes. Cela peut impliquer une mise en balance avec d'autres obligations et impératifs comme le coût ou le poids de telle ou telle obligation sur son destinataire.

20 Constitution de la République Démocratique du Congo telle que modifiée et complétée à ce jour par la loi n¹1/002 du 20 janvier 2011 portant révision de certains articles de la Constitution du 18 février 2006.

21 Loi organique $\mathrm{n}^{\circ} 13 / 011$ du 21 mars 2013 portant institution, organisation et fonctionnement de la Commission Nationale des Droits de l'homme.

22 Articles 211 et 212 de la Constitution de la République Démocratique du Congo telle que modifiée et complétée à ce jour par la loi n ${ }^{\circ} 11 / 002$ du 20 janvier 2011 portant révision de certains articles de la Constitution du 18 février 2006.

23 SAMANTHA BESSON, «L'effectivité des droits de l'homme. Du devoir être, du pouvoir être et de l'être en matière de droits de l'homme ", in Mélanges en honneur de Marco Borghi, 2011, p.63. L'art. 28 de la Déclaration universelle des droits de l'homme du 10 décembre 1948: « Toute personne a droit à ce que règne, sur le plan social et sur le plan international, un ordre tel que les droits et libertés énoncés dans la présente Déclaration puissent y trouver plein effet.». 
De manière tout à fait étonnante, cependant, il existe deux mythes très répandus relativement à la réalisation des droits de l'homme. L'un est que les droits de l'homme sont réalisés de par leur simple existence et ne correspondent pas à des obligations de réalisation et de mise en œuvre. Et le second, qui lui est connexe, est que la non-réalisation d'un droit de l'homme implique qu'il n'existe pas. ${ }^{24} \mathrm{Ni}$ l'une ni l'autre de ces deux affirmations n'est étayée.

Etant donné la nature légale des droits de l'homme, leur mise en œuvre par le droit est une conséquence normative de leur existence en tant que droits de l'homme. Une fois un droit de l'homme reconnu, il génère des obligations à la fois morales et juridiques de mise en ouvre par le droit. ${ }^{25}$ La pratique s'y réfère parfois sous la dénomination d'obligations positives de protection juridique des droits de l'homme.

Il ne faut pas confondre la légalisation ou mise en œuvre légale des droits de l'homme avec leur nature légale et le fait qu'ils doivent être reconnus légalement pour exister en tant que droits de l'homme. ${ }^{26} \mathrm{La}$ reconnaissance légale est une condition de leur existence, alors que la mise en œuvre légale est une conséquence normative de leur existence. De même, il ne faut pas confondre la mise en œuvre légale des droits de l'homme avec leur effectivité. ${ }^{27}$ La mise en œuvre par le droit, comme par tout autre moyen d'ailleurs, ne garantit pas en effet une réalisation complète des droits de l'homme.

Il est important d'indiquer à ce stade que la mise en œuvre des droits de l'homme en République Démocratique du Congo patouille. Madame, le Ministre des droits humains, Marie-Ange Mushobekwa l'a reconnu lorsqu'elle affirme que la République Démocratique du Congo admet des difficultés à mettre en œuvre ses engagements en matière des droits humains parce qu'elle rencontre des obstacles. Elle a pointé du doigt l'insécurité qui se vit sur le territoire et a reconnu la torture dans le milieu carcéral. Ainsi, par la même occasion, elle a plaidé pour une assistance technique des Nations Unies ${ }^{28}$.

\section{B. L'INSECURITE : SOURCE DE VIOLATIONS DES DROITS DE L'HOMME DURANT LE PROCESSUS DE DEMOCRATISATION DANS LA VILLE DE LUBUMBASHI}

\section{Etat des lieux de l'insécurité et de violation des droits de l'homme dans la ville de Lubumbashi}

L'état des lieux de l'insécurité sur la ville de Lubumbashi est déplorable étant donné qu'il y existe de services de sécurité censés protéger les personnes et leurs biens.

24 SAMANTHA BESSON, op cit, p.63.

25 Ibidem, p.64.

26 Idem.

27 Idem.

28 Genève, session du Groupe de travail sur l'examen périodique universel, 07 mai 2019. Disponible sur https://news.un.org/fr/story/2019/05/1042871, page consulté le 02 juin 2019. 
Ce faisant, Il y a lieu de reconnaitre que depuis un certain temps, la ville de Lubumbashi se caractérise par une forme d'insécurité qui ne dit pas son nom et qui cause plusieurs cas de violations des droits de l'homme dont le droit à la vie alors qu'il s'agit d'un Etat qui se veut Etat de droit et déjà bientôt 30 ans engagé dans le processus de démocratisation lequel, présuppose le respect, l'application ou la mise en œuvre des droits de l'homme surtout ceux qualifiées, des droits absolus qui constituant par ce fait el noyau dur.

Certes, il n'est pas aisé de donner un bilan de violations des droits humains suite à l'insécurité dans la ville de Lubumbashi, mais à titre illustratif, il y a lieu d'indiquer, seulement pour cette année 201, depuis son commencement, il a été enregistré 1733 cas d'insécurité dument commentés dont pas moins de 40 cas de vol simple et vol à mains armées accompagnés de tueries ou blessures infligés aux victimes ${ }^{29}$ sans pour autant faire mention à bien d'autres cas passés inaperçus tels que les viols perpétrés en masse par les cambrioleurs armés, la torture infligée au bébé en le mettant dans un congélateur branché au courant pour que ses parents libèrent la somme d'argent exigée les voleurs à mains armées ${ }^{30}$. Ils opèrent sans être inquiétés tout en oubliant que la Constitution de la République démocratique du Congo consacre dans son article 16 Consacre certains droits de l'homme tels que la sacralité de la personne humaine et oblige l'Etat de la respecter et de la protéger; le droit à la vie, à l'intégrité physique ainsi qu'au libre développement de sa personnalité dans le respect de la loi, de l'ordre public, du droit d'autrui et des bonnes mœurs ${ }^{31}$.

Etant donné que l'insécurité a fortement pris de l'ampleur dans la ville de Lubumbashi, le Président de la République, Antoine Tshilombo Tshisekedi était obligé de dépêcher certaines autorités militaires de haut commandement pour éradiquer cette insécurité grandissante. Quelques jours après, lui-même est aussi arrivé à Lubumbashi pour y tenir une grande réunion sur la sécurité en date du 12 avril 2019. Après cette réunion, il a été observé une accalmie mais après quelques temps, c'est toujours l'insécurité qui refait surface. Les gens qui sèment la terreur et l'insécurité sont armés et certaines victimes affirment les avoir vus en uniforme des hommes des troupes.

L'insécurité se vit plus dans certaines communes de la ville de Lubumbashi que d'autres. Les communes considérées comme les zones les plus criminogènes de la ville sont les communes annexes avec $56 \%$ et la commune de Kampemba avec $12 \%$ de cas enregistrés, suivi de la commune de Lubumbashi, Katuba, Rwashi, etc. ${ }^{32}$.

29 Congo durable, «Lubumbashi dénonce l'insécurité à travers une tribune d'expression populaire », in Congo profond, www.congodurable.net/net/2019/03/22.

30 Idem.

31 Article 16 , al. 1 et $2^{\text {ème }}$ de la Constitution du 18 février 2006 telle que modifiée et complétée à ce jour par la loi $\mathrm{n}^{\circ} 11 / 002$ du 20 janvier 2011 portant révision de certains articles de la Constitution.

32 Article 16 , al. 1 et $2^{\text {ème }}$ de la Constitution du 18 février 2006 telle que modifiée et complétée à ce jour par la loi $\mathrm{n}^{\circ} 11 / 002$ du 20 janvier 2011 portant révision de certains articles de la Constitution. 


\section{Les services de sécurité de la ville de Lubumbashi et les violations des droits de l'homme.}

La sécurité nationale fait partie des pouvoirs publics régaliens de l'Etat. Il revient à celui-ci de jouer réellement son rôle de la sécurisation du territoire national. Conformément aux textes qui régissent le pays, la Police nationale, exerce les missions suivantes :

a. veiller à la sécurité et à la tranquillité publique;

b. maintenir et rétablir l'ordre public;

c. assurer la protection rapprochée des hautes autorités ${ }^{33}$.

Il y a lieu de mentionner que la sécurité et la tranquillité publique constituent la première mission des missions de la police nationale congolaise en République Démocratique du Congo. La Police est le principal service public à qui incombe cette mission de protéger les personnes et leurs biens. La Constitution de la République Démocratique du Congo la charge de la sécurité publique, de la sécurité des personnes et de leurs biens, du maintien et du rétablissement de l'ordre public ainsi que de la protection rapprochée des hautes autorités ${ }^{34}$.

Cependant, l'Armée nationale de la République Démocratique du Congo qui a pour mission de défendre l'intégrité du territoire national et les frontières, de manière secondaire, participe aussi, en temps de paix, au développement économique, social et culturel ainsi qu'à la protection des personnes et de leurs biens si nécessité oblige ${ }^{35}$. Bien plus, il y a d'autres services spécialisés qui concourent aussi à la sécurisation de la population dans la ville de Lubumbashi, c'est comme les services de gardiennage (localement appelés Security) qui accomplissent aussi à cette mission.

En effet, bien que ces services existent, ils ne semblent pas accomplir pleinement leur rôle. Les attitudes et les réactions affichées par ces services de sécurité pendant la période d'insécurité inquiètent et planent un doute dans la mesure où leur intervention a toujours été tardive quand bien même ils seraient alertés avant que les voleurs violeurs à mains à armés ne débutent l'opération pour ne pas dire qu'ils n'interviennent que lors que les bandits ont déjà terminé de poser les actes délictueux et violés les droits de personnes ou encore leur intervention est conditionnée par l'achat du carburant par la personne victime qui les appelle alors qu'à l'instant là, les bandits sont à la porte forçant de pénétrer dans la maison. Ceci fait en sorte que les services de sécurité soient accusés de ne pas protéger la population et même d'être complices de ces actes. A propos, le Coordonnateur, Jean-Claude Katende, de l'Association africaine pour la défense des droits de l'homme, une structure des droits de l'homme, a dénoncé dans ses investigations l'impuissance de la Police nationale

33 ANNE-MARIE NSAKA-KABUNDA, «La réforme de la police nationale congolaise et la contribution des partenaires internationaux », s.e, s.d, p. 2, Email : nsakamar@yahoo.fr.

34 Article 182 de la Constitution du 18 février 2006 telle que modifiée et complétée à ce jour par la loi n ${ }^{\circ} 11 / 002$ du 20 janvier 2011 portant révision de certains articles de la Constitution.

35 Article 187 de la Constitution du 18 février 2006 telle que modifiée et complétée à ce jour par la loi n ${ }^{\circ} 1 / 002$ du 20 janvier 2011 portant révision de certains articles de la Constitution. 
face à cette situation en ces termes : « la Police est en train de rater sa mission essentielle. La population se sent abandonnée ${ }^{36}$. Cette période de processus de démocratisation devrait profiter à la République Démocratique du Congo pour se monter déterminer, mieux un Etat qui respecte et applique effectivement des droits de l'homme si elle se veut vraiment un Etat de droit et démocratique.

\section{LES MECANISMES DE SECURITE ET PERSPECTIVES}

\section{Les mécanismes de sécurité}

La République Démocratique du Congo à des règles qui définissent sa politique de sécurité et régissent les services de sécurité. Les premiers mécanismes sont à trouver dans la Constitution de la République Démocratique du Congo du 18 février 2006 telle que modifiée et complétée par la loi $\mathrm{n}^{\circ} 11 / 002$ du 20 janvier 2011 portant révision de certains articles de la Constitution et les autres se trouvent dans les lois spéciales qui organisent et régissent lesdits services de sécurité.

Pour être Policier en République Démocratique du Congo, il faut avoir rempli 11 conditions à savoir : être de nationalité congolaise; être âgé de dix-huit ans au moins et de trentecinq ans au plus sauf pour les exceptions prévues par la présente loi; être célibataire; être de bonne moralité; jouir de la plénitude de ses droits civiques; n'avoir pas été condamné à une peine privative de liberté égale ou supérieure à trois mois de servitude pénale principale ni révoqué de l'administration ou d'une entreprise publique; n'appartenir à aucun parti politique ni regroupement politique, à moins d'avoir démissionné préalablement au dépôt de sa candidature; fournir un extrait du casier judiciaire en cours de validité; fournir les titres scolaires requis; être reconnu physiquement et psychiquement apte et être classé en ordre utile au concours de recrutement ${ }^{37}$.

Mais force est de constater que ces conditions ne sont pas scrupuleusement respectées dans le recrutement des éléments dans la Police nationale. Le recrutement se fait sur base d'autres critères inconnus qui ne peuvent pas permettre d'avoir une Police efficace en qualité pour accomplir sa mission de sécuriser les personnes et leurs biens. Il convient de croire que la recrudescence de l'insécurité en République Démocratique du Congo est même liée au mauvais recrutement des éléments de l'ordre, au non encadrement des jeunes congolais sans emplois mais surtout à l'inapplication de la loi qui implique aussi l'impunité.

En outre, la population accuse la Police de la léthargie et de la justice de complaisance en disant que : « La léthargie et la complaisance de justice qui feint de punir les bandits sans vraiment les mettre hors d'état de nuire. Aux autres de décrier le manque de moyens

36 «Insécurité à Lubumbashi : le député Eliezer Thambwe saisit le gouvernement », mardi 9 avril 2019, https://actualite.cd/index.php/2019.

37 Article 17 de la Loi ${ }^{\circ} 13 / 013$ du 1er juin 2013 portant statut du personnel de carrière de la police nationale congolaise. 
dont souffriraient la police et les chefs de quartiers notamment. La complicité de la police avec les malfrats serait une réalité dans plusieurs parties de la ville».

C'est inconcevable lors que le niveau de criminalité, comme l'a affirmé, Jeff Mbiya, acteur de la Société civile du Haut-Katanga, dépasse la capacité opérationnelle des éléments de sécurité et renseignement ${ }^{38}$.

A ce sujet, une préoccupation demeure sans réponse, c'est celle de savoir si cette insécurité est due à l'impuissance de riposte qu'à la Police nationale ou c'est un problème de manque de moyens nécessaires mis à la disposition de la Police par les autorités qui ne permettent pas à cette dernière d'accomplir correctement sa lourde tâche ou encore c'est de la complicité sans les chaines de commandement.

Il ne suffit pas seulement que l'autorité de la ville reconnait que la ville n'est pas calme suite à l'insécurité et ses conséquences entre autres, le vol, viol, torture, meurtre, l'inaction de l'autorité comme l'a fait Madame Laurianne MWEWA, adjointe au maire de la ville lors d'une tribune d'expression organisée le jeudi, 21 mars 2019, il faut chercher les solutions y relatives. Pour elle, c'est une occasion à saisir pour demander à la population via les cadres de base de participer aussi à la gestion de son milieu par des propositions pouvant améliorer la bonne gouvernance urbaine ${ }^{39}$.

De ce qui précède, il sied d'affirmer que les mécanismes institutionnels, les lois et règlements existent mais ne s'appliquent pas. La Police nationale n'accomplit pas ses missions comme prévues par la Constitution ${ }^{40}$, les lois et règlements de la République. Elle devient de plus en plus politisée ${ }^{41}$ alors que la Constitution veut que la Police nationale soit apolitique, au service de la Nation congolaise et ne soit pas détournée à des fins propres ${ }^{42}$. Ce qui a pour conséquence, les violations massives de droits de l'homme dans la République démocratique du Congo en général et dans la ville de Lubumbashi en particulier.

Il n'y a pas de paix, de démocratie, de développement ni respect des droits de l'homme sans sécurité. C'est-à-dire, la démocratie et le développement engendrent la sécurité et la

38 JEFF MBIYA cité par Patrick KASONDE, art cit.

39 JEFF MBIYA cité par Patrick KASONDE, art cit.

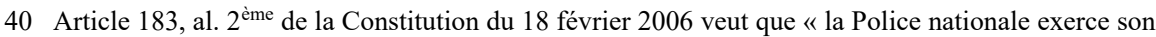
action sur l'ensemble du territoire national dans le respect de la présente Constitution et des lois de la République » et l'Article 55de la Loi n $13 / 013$ du 1er juin 2013 portant statut du personnel de carrière de la police nationale ajoute que « le Policier ne doit se livrer à aucune activité contraire à la Constitution, aux Lois de la République et aux Règles régissant sa profession ».

41 Lorsque que le député national ELIEZER THAMBWE été allé voir le vice premier ministre en charge de l'intérieur et sécurité BASILE OLONGO pour lui faire part de la situation d'insécurité dans la ville de Lubumbashi, le vice premier ministre a pu évoquer quelques implications politiques, dans cette situation. "Insécurité à Lubumbashi : le député ELIEZER THAMBWE saisit le gouvernement », mardi 9 avril 2019, https://actualite.cd/index.php/2019.

42 Article 183 de la Constitution du 18 février 2006 telle que modifiée et complétée à ce jour par la loi n $11 / 002$ du 20 janvier 2011 portant révision de certains articles de la Constitution du 18 février 2006. 
paix $^{43}$. Construire ou bâtir un Etat de droit nécessite une réforme des institutions publiques, y compris celles opérant dans le secteur de la sécurité comme la Police nationale ${ }^{44}$, Armée, Agence nationale de Renseignement et autres services de sécurité spécialisés. Parmi les attributions originelles d'Etat, se trouve aussi celle de la sécurité de personnes et leurs biens. Il est inadmissible dans un Etat de droit que la population se prenne en charge dans le secteur de la sécurité mais peut participer par sa collaboration à ce secteur. C'est à l'Etat Congolais que revient cette obligation de sécuriser le territoire national et la population congolaise.

Ainsi, suggérons que les nouvelles méthodes de renforcement de confiance entre les protecteurs (services publics de sécurité) et les protégés (la population) soient prises, car la population devient méfiant vis-à-vis de la Police, voire l'Armée. Que la République Démocratique du Congo renforce sa politique sur la sécurité en adoptant des mécanismes efficaces notamment dans l'organisation de la sécurité publique, dans le recrutement des éléments de l'ordre. La sécurité passe par une bonne administration d'un Etat et de manière particulière par la justice comme cela doit l'être dans un Etat de droit.

Le recensement de la population s'avère très important pour identifier et maîtriser le flux de la population dans la ville de Lubumbashi. Il revient aux chefs de quartiers d'identifier et d'enregistrer avec précision d'adresse complète de ceux qui viennent et qui repartent parce que la recrudescence de l'insécurité qualifiée d'une autre face des violations des droits de l'homme a pris de l'ampleur avec la masse de la population qui vienne d'autres coins du territoire de la République Démocratique du Congo. C'est connu de tous.

\section{CONCLUSION}

Les droits de l'homme comprennent notamment, l'interdiction de la discrimination, le droit à la vie, l'interdiction de la torture et de traitements inhumains, l'interdiction de l'esclavage, la liberté d'opinion et d'expression, la liberté de pensée et de religion, la liberté d'association et de réunion, la protection de la sphère privée et de la vie de famille, le droit à une procédure judiciaire équitable, la protection de l'individu contre l'exploitation et à lui donner le droit de participer à la richesse sociale, le droit au travail, le droit à des conditions de travail correctes et acceptables, le droit à la sécurité sociale, le droit à la protection de la famille, de la maternité et des enfants, le droit à la santé etc...

Ces libertés et droits fondamentaux ont un caractère juridique obligatoire depuis la Déclaration universelle des droits de l'homme de 1948 et bien d'autres instruments juridiques internationaux relatifs aux droits de l'homme déjà ratifiés par la République Démocratique du Congo. Ces droits et libertés sont censés être appliqués effectivement dans tout Etat qui se veut Etat de droit et gagné dans le chemin de la démocratie. Le processus de démocratisation ne peut constituer en rien un obstacle pour l'application des droits de l'homme. C'est 
plutôt un moment propice un Etat qui se veut démocratique de valoir sa capacité d'appliquer ces droits. L'Etat de droit présuppose l'application, le respect des droits de l'homme et la démocratie.

L'insécurité dans la ville de Lubumbashi est à déplorer ces jours et est à la base de beaucoup de cas des violations des droits de l'homme. Elle est ici qualifiée d'une autre face des violations des droits de l'homme. Et pourtant, ces droits ne doivent pas souffrir d'application quelle que soit la période que traverse la République Démocratique du Congo.

En principe, c'est pendant cette période de processus de démocratisation que la République Démocratique du Congo devrait se montrer déterminée dans l'application des droits de l'homme si elle se veut vraiment un Etat de droit et démocratique.

La recrudescence est un fait lié à une mauvaise politique sécuritaire mise en place et à l'inapplication des lois prévues dans ce secteur. Le mauvais recrutement des éléments de la sécurité et le non encadrement des jeunes congolais sans emploi ni activité accentuent cette situation. Il en est de même de l'Administration publique politisée et en déconfiture. Et pourtant, dans un Etat dit Etat de droit, c'est l'Administration qui est le bras séculier d'un Etat pareil.

Un Etat de droit, c'est celui qui applique la loi quand il faut l'appliquer et ce, sans interférence politique de qui que ce soit.

Si les droits de l'homme sont violés suite à l'insécurité dans cette ville de Lubumbashi, c'est parce que les instances habilitées à sanctionner ne jouent pas correctement leur rôle. Il faut punir les violeurs de ces droits conformément à la loi.

La non application effective de la loi et les interférences politiques en faveur des violeurs des droits de l'homme seraient à la base de la défaillance de l'Etat d'assurer la protection des droits de l'homme (tels que le droits à la vie des citoyens, le droit à la sécurité sociale, le droit à la protection de la famille...), la sécurité de ses citoyens et de leurs biens.

De ce fait, il convient de suggérer que la République Démocratique du Congo renforce sa politique sur la sécurité en adoptant des mécanismes efficaces dans l'organisation de la sécurité publique, le recadrage de ses techniques sécuritaires, le recrutement des agents de l'ordre doit être objectif et sérieux et non de remplacement du personnel de l'ordre par fantaisie ou suite au décès d'une personne membre de la famille pour ne pas perdre le numéro matricule de la fonction publique. La sécurité passe par la bonne administration de la justice comme cela doit l'être dans un Etat de droit.

Terminons cette réflexion en rappelant que son est non seulement de décrier l'insécurité qui constitue une nouvelle facette des violations des droits de l'homme, mais aussi d'interpeler davantage la conscience de l'autorité pour qu'elle s'implique afin de l'éradiquer et de militer que la mise en œuvre de ces droits soit effective. 


\section{REFERENCES BIBLIOGRAPHIQUES}

\section{TEXTES DES LOIS}

Constitution de la République Démocratique du Congo telle que modifiée et complétée à ce jour par la loi n $11 / 002$ du 20 janvier 2011 portant révision de certains articles de la Constitution du 18 février 2006.

Déclaration universelle des droits de l'homme du 10 décembre 1948.

Loi organique $n^{\circ} 13 / 011$ du 21 mars 2013 portant institution, organisation et fonctionnement de la Commission Nationale des Droits de l'homme.

Loi n $13 / 013$ du 1er juin 2013 portant statut du personnel de carrière de la police nationale congolaise.

\section{OUVRAGES}

ANDRIEN MULUMBATI NGASHA, Manuel de sociologie générale, Lubumbashi, éditions Africa, 2010.

DANIEL LOCHAK, Les droits de l'homme, éditions La Découverte, Collection : Repères, 2018.

JEAN-MARC DE LA SABLIERE, Indispensable ONU, éditions PILON, Paris, 2017.

NGONDANKOY NKOY-EA-LOONGYA, Droit congolais des droits, éditions Academia-Bruylant, Paris, 2004.

PIERRE FELIX KANDOLO ON'UFUKU WA KANDOLO, Guide Kandolo: Méthodes et règles de rédaction d'un travail de recherche en droit, éditions Universitaires Européennes, Mauritius, 2018.

RENE-JEAN DUPUY, Une œuvre au service de l'humanité, UNESCO, Paris, 1999.

RIVERO JEAN, Libertés publiques, Manuel, éditions PUF, 1973.

ROCHE JEAN et POUILLE ANDRE, Libertés publiques et droits de l'Homme, $13^{\mathrm{ème}}$, édition, 1999.

RUSEN ERGEC, Protection européenne et internationale des droits de l'homme, Bruxelles, Bruylant, 2004.

\section{ARTICLES}

ANNE-MARIE NSAKA-KABUNDA, « La réforme de la police nationale congolaise et la contribution des partenaires internationaux », s.e, s.d,, Email : nsakamar@yahoo.fr.

CESAR NKUKU KHONDE (dir.), «Droits de l'homme au Congo colonial : exposé et analyse de quelques faits et témoignages des abus », La République Démocratique du Congo : Les droits humains, les conflits et la construction/destruction de l'Etat, édition Fondacio Solidaritat UB et Inrevés, Barcelone, 2009.

DELPHINE POLLET-PANOUSSIS, « La Constitution congolaise de 2006 : petite sœur africaine de la Constitution française ", in Revue française de droit constitutionnel, Presses Universitaires de France, 2008/3 $\mathrm{n}^{\circ} 75$.

FABRICE PARFAIT OUMBA, Les mécanismes de contrôle et de garantie des droits de l'homme. Disponible sur https://hal.archives-ouvertes.fr/cel-01319645, Submitted on 21 may 2016, page consultée le $05 / 06 / 2019$. 
PIERRE-FELIX KANDOLO ON'UFUKU WA KANDOLO, De l'exercice des droits et libertés individuels et collectifs comme garantie d'une bonne gouvernance en Afrique noire : Cas de la République Démocratique du Congo, Université de Nantes/Université de Lubumbashi, Campus numériques, $3^{\text {ème }}$ cycle des Droits fondamentaux. Disponible sur https://www.mémoireonliine.com, page consultée le 02 juin 2019.

VERONIQUE CHAMPEIL-DESPLATS, « Démocratie, droits de l'homme et contrôle de constitutionnalité chez Norberto Bobbio », in La démocratie, entre multiplication des droits et contre-pouvoirs sociaux, éditions Kimé, collection : Nomos et Normes, 2012.

SAMANTHA BESSON, "L'effectivité des droits de l'homme. Du devoir être, du pouvoir être et de l'être en matière de droits de l'homme », in Mélanges en honneur de Marco Borghi, 2011.

\section{WEBOGRAPHIE}

Https://www.vie-publique.fr/decouvertinstitutions/institutions/approfondissements/qu-est-ce-que-etat-d roit.html, page consultée le 06 juin 2019.

Https://news.un.org/fr/story/2019/05/1042871, page consulté le 02 juin 2019.

Http://ww.congodurable.net/net/2019/03/22, page consultée le 25 mai 2019.

Https://actualite.cd/index.php/2019, page consultée le 18 mai 2019. 